Title:

\title{
Salvage Local Treatments After Focal Therapy for Prostate Cancer
}

\section{Authors}

Giancarlo Marra ${ }^{1,4}$, Massimo Valerio ${ }^{2,}{ }^{\dagger}$, Mark Emberton $^{3}$, Axel Heidenreich $^{4}$, Juanita M. Crook ${ }^{5}$, Alberto Bossi ${ }^{6}$, Louis L Pisters ${ }^{7}$

${ }^{\forall}=$ the two authors contributed equally and share the first authorship

\section{Institutions}

1= Department of Urology, San Giovanni Battista Hospital, Città della Salute e della Scienza and University of Turin, Turin, Italy

2 = Department of Urology, Centre Hospitalier Universitaire Vaudois, Lausanne, Switzerland

3 = Division of Surgery and Interventional Science, University College London, London, UK

4 = Department of Urology, Uro-Oncology, Robot-Assisted and Reconstructive Urologic Surgery, University Hospital Cologne, Cologne Germany

$5=$ Department of Surgery, Division of Radiation and Developmental Radiotherapeutics, University of British Columbia, BC Cancer Center for the Southern Interior, Kelowna, BC, Canada

6 = Department of Urology, Gustave Roussy Cancer Institute, Villejuif, France

7 = Department of Urology, MD Anderson Cancer Center, TX, USA

\section{Corresponding Author}

Giancarlo Marra

e-mail: drgiancarlomarra@gmail.com

Institution:

Department of Urology

San Giovanni Battista Hospital

Città della Salute e della Scienza and University of Turin

C.so Bramante 88/90

10100 Turin, Italy

Phone: +390116336594

Fax: +390116335707

\section{Abstract}


Context: Whether focal therapy (FT) for prostate cancer (PCa) jeopardizes outcomes of salvage treatments is a matter of debate awaiting to be resolved.

Objective: To review the literature investigating oncological and functional outcomes as well as complications of available treatment options for recurrent or residual disease after primary FT.

Evidence Acquisition: We performed a non-systematic web search using pubmed, including all articles aiming at assessing relevant outcomes of salvage local treatment after FT failure. Web search was implemented by manual search. When no evidence could be extracted in the FT domain, records dealing with recurrence after whole-gland ablation were considered.

Evidence Synthesis: four retrospective series assessed salvage treatments after FT failure evaluating cases of radical prostatectomy (RP) and re-do ablation (included patients $n=12-22$ ). The studies' quality was low with a high risk of bias. Other options are radiation therapy (RT) and whole gland or focal re-do ablations which, however, have only been described after whole-gland ablation. With some exceptions, including sexual function for RP, overall complications, oncological and functional outcomes do seem acceptable not being much worse than those of a primary setting. Important limitations include low level of evidence and absence of standardized criteria for FT, salvage treatment and FT failure.

Conclusions: Current evidence shows acceptable outcomes of post-FT salvage options, although this is based on retrospective data. While it seems that FT has minimal impact on salvage treatment results, prospective controlled studies are needed to confirm this preliminary data.

Patient Summary: We performed a literature search to determine which treatment options are available to cure PCa in case of FT failure and what are their outcomes. There are various options including RP, re-do whole-gland and focal ablation and RT. Overall cancer control, impact on urinary and sexual function and complications seem slightly worse but do not yield any marked differences compared to primary treatments. Nonetheless, only a few studies analyzing these treatments are available. This does not allow any definite conclusion and/or recommendations but only favors the hypothesis FT marginally jeopardizes subsequent salvage therapy results. High quality studies are awaited to confirm or not these findings.

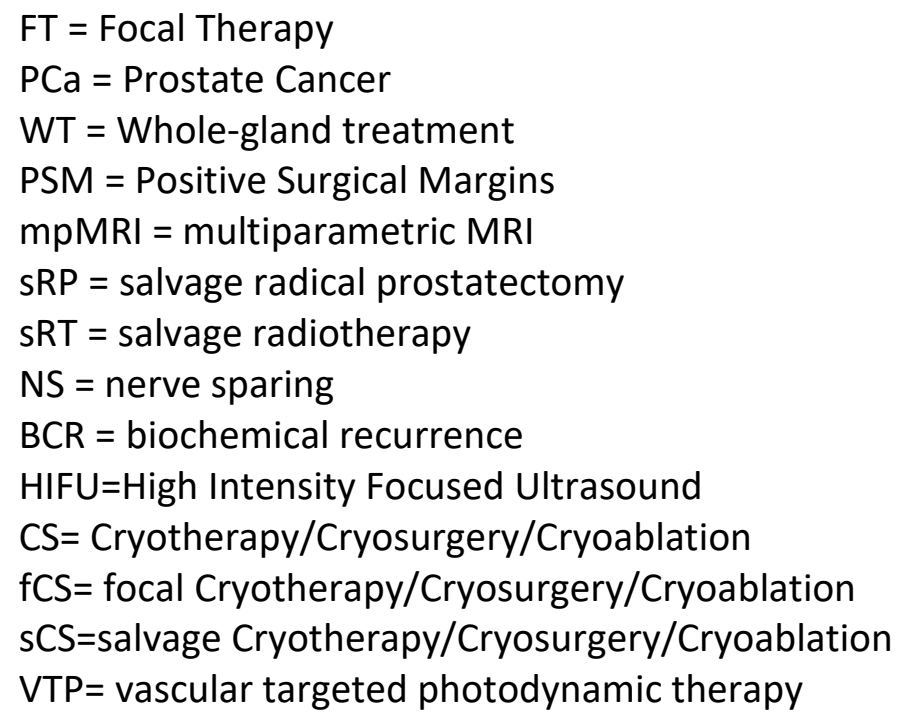

\section{Manuscript}




\section{Introduction}

Focal therapy (FT) for prostate cancer (PCa) has been proposed as a minimally invasive option to treat localized disease in men qualifying for such approach. The aim is to reduce side effects related to radical treatment whilst maintaining the oncological benefit of local treatments [1-3]; however, the ideal eligibility criteria in FT are not well defined. Although FT is not generally considered a standard of care option, short- to medium-term results are now available, showing acceptable oncological outcomes and low toxicity $[2,4]$.

Several concerns remain. First, PCa is multifocal in the majority of the cases, as documented by approximately $80 \%$ of radical prostatectomy (RP) large series; second, despite recent improvements through the use of multiparametric MRI (mpMRI) based targeted biopsies, inappropriate patient selection may result in disease progression, and thus worse outcome than initial radical treatment; third, no long-term oncological results based on prospective controlled cohorts are available at present [1,3-6]. Finally, as per other non-surgical whole-gland treatment (WT) modalities, FT may jeopardize subsequent salvage treatment outcomes in case of PCa recurrence [5].

The latter issue in particular, will likely become crucial. Although FT should still be considered experimental, it is considered attractive by patients and by the large urological community as the likelihood of preserving genito-urinary function is better than with standard radical treatment, and the chances of disease progression appear acceptable in the mid-term [7]. Short- to medium-term FT series do yield up to a $17 \%$ rate of clinically significant PCa persistence and/or recurrence, with around $28 \%$ needing additional local treatment $[1,2]$. As a consequence, the outcome of salvage treatments remains a key issue to be resolved in order to evaluate the trade-off between initial focal or radical treatment. If an initial focal treatment does not negatively impact subsequent treatment, then many would prefer this approach; however, if an initial FT would have a major detrimental effect on secondary treatments, then it should be reserved to very well selected patients in whom the chances of recurrence are extremely low.

Currently, several salvage options for FT failure can be envisioned. For low-risk disease, active surveillance may be appropriate. In case of intermediate- to high-risk PCa, active treatment with curative intent should be regarded as the standard. This includes: i) re-do treatments, using the same or different energy sources compared to the first line setting,; ii) salvage RP (sRP); or iii) salvage radiotherapy (sRT), either whole gland or focal, external beam or brachytherapy.

From an oncological perspective, selection of more resistant PCa clones may result in more aggressive tumor biology at recurrence; from a functional perspective, well-documented phenomena including neo-angiogenesis, necrosis and tissue scarring, may alter anatomical landmarks, surgical planes and healing potential, possibly increasing complication risks and impairing recovery [8-13]. Whilst these effects are well documented following whole-gland nonsurgical treatments, little evidence exists following FT, which, by definition, aims to reduce collateral effects of radical treatment.

In this paper, we aimed to perform a literature review, with the objective of reporting the impact of prior focal ablation on complications, functional and oncological outcomes of salvage treatments for recurrent or residual PCa.

\section{Methods}


A non-systematic web search was performed independently by two authors (G.M. and M.V.) using Pubmed on September 5th 2018, with no time restrictions. The words "focal therapy", "prostate cancer" and "recurrence" were put together using the boolean operator "AND". We included all relevant English language articles dealing with salvage treatments following PCa recurrence after primary FT. Additional articles dealing with recurrences after whole-gland ablation were also considered when no evidence was found for primary FT. Web search was implemented by manual search of included articles references and by senior authors' consultation. When possible continence was classified according to the number of pads/day; erectile function according to the IIEF-5 or according to patients' reports of preserved erections (spontaneous or with PDE-5 inhibitors) versus others; complications according to the Clavien-Dindo classification [14] and the EAU guidelines [15].

\section{Results}

We found 3 series reporting on SRP after FT. Only one series reported re-do ablation results and no series investigated RT after FT; for the purpose of completeness, we reported the results of the main studies on re-do ablation and salvage RT after whole-gland radiotherapy and/or ablation. We found no series relating to outcomes of active surveillance, watchful waiting and ADT after initial FT. Evidence concerning their use in a post-FT setting remains mainly based on expert groups' experience (no published series available) $[16,17]$. Since these are non-interventional and/or do not have a curative aim and an overall survival endpoint will require many more years to allow results, their evaluation is beyond the scope of the present review.

\subsection{Salvage Re-Ablation}

Focal or whole-gland ablation using the same or different energy sources are possible options and have been used after failure in FT series, ranging up to 33\% [1]. Some expert groups support re-do procedures based on their own experience. Nonetheless, results from these institutions have not yet been published $[16,17]$ and direct evidence on re-ablation efficacy following FT remains scarcely documented.

Currently two energy sources have been assessed as re-do options almost exclusively following whole-gland ablation.

Chang and colleagues reported outcomes of salvage whole-gland CS for local recurrence performed in 12 men at a median of 7 months (range 6-99) after focal CS (fCS). At a mean followup of 33.5 months, seven men were disease-free, three underwent ADT because of disease progression and the remaining two underwent another cycle of $\mathrm{SCS}$, being disease-free at subsequent controls. No major complications were reported. One man who underwent repeat CS developed mild (1-2pads/day) incontinence; of the two potent patients before the procedure, none experienced permanent erectile dysfunction [18]. This work is currently the sole study specifically assessing the outcomes of a re-do treatment after FT. No large series of salvage CS after FT or whole-gland ablation specifically addressing its functional and oncological outcomes are available.

Salvage CS outcomes were also assessed by a large cohort from the USA including recurrent cases after primary RT ( $n=259)$, low-dose rate brachytherapy $(n=49)$, or CS $(n=20)$. At a median follow-up 
of 47.0 (1.6-203.5) months, $n=160$ (49\%) experienced recurrence and $n=108$ men (33\%) died, $\mathrm{n}=35$ (10.7\%) due to PCa (10-yr Disease-free, OS and CSS being 35\%, 45\% and 79\% respectively). Complications were relatively infrequent, occurring in $n=46(14 \%)$ men with urethral strictures $(n=15)$ being the most frequent adverse event. Six cases of fistula were recorded. Seven men had severe incontinence and underwent subsequent surgical treatment [19]. Interestingly, a subgroup of $n=55(16.7 \%)$ men received fsCS, including 5 cases of primary focal CS (results not reported separately). Ten-year disease-free survival was similar to whole-gland sCS being $42 \%$ so as for CSS being $83 \%$; surprisingly 10 -years OS was much higher in the fsCS group being $81 \%$. Again, serious complications were uncommon. Nonetheless, three cases (5\%) of fistula were noted. As possible explanations authors stated these cases occurred at the beginning of their fsCS experience with two of them also receiving two fsCS sessions each.

Re-do HIFU is another possible option. Berger and co-workers specifically investigated the effect of re-do whole-gland HIFU compared to a single whole-gland HIFU treatment in terms of complications and functional outcomes [20]. Of 359 included men, 130 (36.2\%) underwent one or more redo HIFU ( $n=19$ two redo-sessions and $n=1$ three redo-sessions), the remaining a single whole-gland HIFU. No major baseline differences were identified with the exception of higher Gleason score prior to re-do HIFU ( $p=0.004)$. Re-do HIFU yielded higher biochemical recurrence (BCR) (44.8\% vs $26.4 \%)$, possibly related to the higher Gleason scores. Intra- and post-operative complications, including the need for additional procedures, were similar amongst the two cohorts with the exception of UTI, less frequent after re-do treatment $(p=0.009)$. Moreover, the re-do group recorded shorter treatment time $(p<0.001)$, hospital stay $(p=0.002)$ and catheterization time $(p=0.001)$ compared to first HIFU. After re-do HIFU, there was an increase in urinary leak ( $p$ $<0.001)$ although this did not translate into a statistically significant increase in those using pads $(p=0.07)$. No significant changes in sexual function $(p=0.9)$ were recorded [20].

Similar findings were reported by others evaluating 223 men undergoing a single- and 49 men undergoing two or more re-do HIFU sessions. Re-do procedures yielded shorter operating time and similar complications. Incontinence risk following two HIFU session was overall increased compared to baseline $(12.2 \%, p=0.024)$. However, this was rather related to first-line whole-gland HIFU as re-do HIFU did not yield a statistically significant impact on continence $(p=0.266)$.

On the contrary, an increased erectile dysfunction risk was present compared to baseline $(55 \%, p<0.01)$ and persisted when comparing erectile function before- and after second HIFU $(p=0.039)[21]$.

Recently, Guillaumier and colleagues published intermediate-term results of focal HIFU in a prospective multicenter cohort of 599 men. At a median follow up of 56 months (IQR 35-70), $18.7 \%(n=112)$ and $1.5 \%(n=9)$ had one or two re-do HIFU respectively [2]. Unfortunately, functional and oncological results of re-do procedures were not specifically addressed leaving the specific impact of re-do HIFU sessions after FT not determined.

Irreversible electroporation and high-dose rate focal brachytherapy have also been described in a salvage context and do seem feasible after FT failure. However, evidence is scarce and warrants further assessment $[22,23]$.

\subsection{Salvage Surgery}


To date, three series reported the outcomes of sRP after first-line focal treatments.

Lebdai and colleagues investigated the outcomes of 19 men undergoing radical prostatectomy for PCa recurrence in 8 French institutions ( $n=12$ open, $n=5$ robotic; $n=2$ laparoscopic) after focal photodynamic therapy. Nine patients (47\%) had positive margins (PSM) with six needing subsequent sRT. At a median follow-up of 10 (1-46) months after sRP, PSA was undetectable in 16 men (84\%). The dissection was considered difficult in seven cases (37\%) at the posterior and lateral sides of the prostate due to local fibrosis, not allowing nerve sparing (NS) procedures. Continence was achieved post-operatively in the majority $(n=13 ; 68 \%)$ ); five $(26 \%)$ had mild incontinence needing $\leq 1 \mathrm{pad} / \mathrm{day}$ and one man (5\%) had severe incontinence needing $>2$ pads/day. Complications were acceptable with $n=1$ man having accidental intra-operative ureteral transection during lymphadenectomy, $n=1$ having a pelvic hematoma treated by radiological evacuation and no other major (Clavien $\geq \mathrm{III}$ ) complications. Median hospital stay, catheterization time and blood loss were 7days, 7days and 400cc respectively. Interestingly, authors found prior bilateral vascular targeted photodynamic therapy (VTP) treatment was significantly correlated with PSM $(p=0.003)$ and to a "difficult" dissection $(p=0.009)$ [24]. While this series is heterogeneous by nature with relevant confounders - such as surgical expertise, low number of cases per institution, heterogeneous surgical technique and ablation - it does show that the procedure is feasible, has acceptable complication rate, although the surgical and genito-urinary outcome might be compromised.

The other two available series belong to the same institution and thus overlap of some patients is likely to have occurred.

In the first work, Linares and colleagues included 28 men undergoing laparoscopic or robotassisted SRP, half of whom had FT as primary treatment [25]. One versus three patients had PSM in the FT versus WT, all having extracapsular disease. Importantly, pathological stage was worse in the WT group ( $p=0.008)$. At median follow-up of 62 months $n=7$ FT versus $n=4$ WT had $B C R(p=0.8)$ with one cancer-related death being recorded in a pT4a Gleason 4+5 R1 disease (WT group). No significant differences were found at 12 months in continence with one patient (7\%) having severe incontinence ( $>2$ pads/day) and seven (50\%) being fully continent in the FT group. No intraoperative complications occurred and overall major complications were $n=3$, including $n=1$ myocardial infarction and $n=2$ urinary leaks. No differences were noted in overall complications $(p=0.68)$, mean blood loss ( $p=0.58)$ and median hospital stay $(p=0.36)$ being $n=7,550 c c$ and 4 days respectively in the FT group. Interestingly, those who underwent FT as primary treatment had shorter operating time (133 \pm 21 vs WT $176 \pm 34 \mathrm{~min}, p=0.001)$ [25].

In 2018, Nunes-Silva et al published a second paper including 23 men undergoing robotic sRP after FT who were compared with 44 primary robotic RP, selected through a matched-pair analysis from 2,775 cases [26]. Baseline features were comparable (pathological Gleason score $(p=0.256)$ and stage $(p=0.705)$; pre-operative PSA $(p=0.691))$. Surgical margins were positive in $n=1$ sRP $(4 \%)$ vs 8 men $(18 \%)$ in the primary RARP although this was not significant $(p=0.253)$. Similarly, BCR rates were comparable amongst the groups $(p=0.426)$ occurring in $31.82 \%$ of sRP. However, sRP showed a shorter median time to BCR $(p=0.0001)$ and a lower probability of BCR-free survival $(56.3 \%$ vs $92.4 \%$ at 2 years, $p=0.001)$ yielding a $4.8(p=0.004)$ increased $B C R$ risk compared to primary $R P$.

Pad-free probability rate was comparable with $73 \%$ vs $76.5 \%$ being continent at 2 years ( $p=0.8$ ). Preoperatively potent patients undergoing unilateral or bilateral nerve sparing had lower mean 
IIEF-5 after sRP ( $p=0.008)$. Overall complications did not differ $(p=0.742)$, although high-grade complications $(n=3)$ were found only in the SRP group.

Operative time $(p=0.625)$, hospital stay $(p=0.999)$, catheterization time $(p=0.637)$ and EBL $(p=0.596)$ did not differ significantly [26].

A pelvic lymph-node dissection was performed inconsistently in these two series (29\% [25] and $31.8 \%$ [26], respectively) whilst a higher percentage of patients underwent the procedure after primary VTP (78.9\%) [24]. The latter study also described the sole case of N1 disease being recorded.

\subsection{Salvage Radiation Therapy}

Currently, no series have reported the use of sRT after FT failure. Nonetheless, several authors reported retrospectively the outcomes of small SRT series after whole-gland HIFU and/or cryotherapy (CS).

Following a feasibility study [27], Riviere and colleagues reported outcomes of the largest sRT series, including 100 men with histologically proven recurrence and at least 1 year follow-up, being treated with 72 Gy EBRT after 1 or 2 HIFU sessions (17 of whom received adjuvant ADT) [28]. At a median follow up of 36.5 months, 15 (15\%) patients who received EBRT failed treatment (5year progression-free survival 72.5\%) and 10 (10\%) died, one of whom due to metastatic PCa. High grade toxicities were rare and included 3 acute urinary retention requiring endoscopic treatment, one chronic painful retention requiring urinary diversion and one death due to multi-organ failure after a haemostatic cystectomy. Incontinence did not change significantly (28\% before- to $32 \%$ post-sRT; $p>0.05$ ). Erectile dysfunction severity increased following primary HIFU and again after SRT.

These results are supported by the second largest post-HIFU SRT series, also yielding the longest follow-up to date (mean 50 months). This Italian group analysed 76 Gy 3D-Conformal sRT outcomes after one $(n=17)$ or two $(n=7)$ HIFU sessions. Four men $(16.6 \%)$ received a pelvic radiotherapy field (including bilateral ileo-obturator chains) and seven (29\%) received adjuvant ADT. BCR occurred in 4 men (16.6\%). High-grade toxicity was relatively rare including 3 acute genitourinary cases (grade III $n=2$; grade IV $n=1$ ) and 5 grade III late genitourinary ( 3 severe frequency and dysuria; 1 frequent hematuria) or bowel toxicity (1 rectal morbidity). At 1-year, two men developed permanent post-sRT incontinence (8\%) [29]. Another recent series including six patients also showed acceptable functional and oncological results together with relatively low toxicity concerning SRT post-whole gland HIFU [30].

The largest series of sRT post-CS evaluated men undergoing sEBRT (mean dose 62.9 Gy) following one $(n=39)$, two $(n=8)$ or three $(n=2)$ CS sessions between 1990 and 1999. Four patients received additional pelvic irradiation (8\%). At a median follow-up of 32 months, $61 \%$ were free from BCR. No acute high-grade toxicities were recorded whilst 2 experienced subacute late morbidity all being managed conservatively ( 1 urethral stricture at 10 months; 1 proctitis at 2 months). No new onset incontinence occurred [31].

Hepel et al also reported the outcomes of 16 men treated with 72 Gy full-dose IMRT either adjuvantly after focal CS ( $n=3)$ or in a salvage context after local failure of standard whole-prostate 
CS ( $n=13)$ from 1997 to 2007. At a median follow-up of 33 months BCR occurred in four patients (25\%). No major toxicities were recorded [32].

Other small case series investigated sRT after CS using dose-escalated IMRT [33], IMRT [34], EBRT [35] and CyberKnife robotic stereotactic body RT [36], all yielding promising results. [36]

Finally, Holtzman et al described 74 Gy proton beam sRT in 21 men recurring after either HIFU $(n=9)$ or CS $(n=12)$. Two cases showing higher risk of positive node were also treated by additional 46 Gy pelvic IMRT followed by a proton boost to the prostate and seminal vesicles. Three-year BCR-free survival was $77 \%$ with 5 men having recurrence ( 1 biochemical only, 1 local, $n=2$ regional and 1 distant -4 having GS =8). Cumulative 3 -year grade 3 toxicity was $17 \%(95 \% \mathrm{Cl}: 5-42 \%$; $=3$ CTCAE v4). Sexual and urinary functions showed a mild decline only at 12 months [37].

No studies concerning SRT after ablative energy sources other than HIFU or CS are currently available.

\section{Discussion}

This review shows that the outcomes of salvage treatments in case of FT failure remain basically to be further elucidated. The literature over second ablation and SRT is scarce and refers mainly to re-do after primary whole-gland treatments showing that the complication rate and the functional outcome are encouraging. There are few reports of sRP after FT suggesting that, although some cases may be technically more challenging, oncological and functional results are promising and do not seem to markedly differ from first line RP.

Despite the unresolved debates around FT in PCa, its implementation in clinical practice is rapidly growing [5] and is likely to further increase in the in the coming years. In 2014 a systematic review included approximately 2,000 patients being treated with tissue sparing approaches; two years later another systematic review on the same subject already reported outcomes for more than 3,200 men $[1,4]$. Medium-term FT outcomes have been recently published suggesting acceptable results in a prospective multicenter cohort of 599 men including nine centers in the UK offering FT off-trial under local governance and insurance agreements [2]. The wide European urological community is highly interested in this option as shown by a recent survey in which $52 \%$ of participants stated they suggest FT to patients [7]. Also, 65\% confirmed that they had access to FT either in their hospital (44.6\%) or in their region (55.4\%). While the field is quickly moving forward, a new issue is likely to become more relevant: the management of men failing primary FT. If the rate of secondary treatment is estimated to be around $20-25 \%$ over 5 years, and the number of men being treated with FT is increasing, this means that a substantial group of patients will require further treatment in the near future.

Currently, in a post-FT setting, RP seems an acceptable oncological option with the prior FT having little impact compared to primary surgery. PSM are reported in $20 \%$ and BCR in $33 \%$ with no clinical PCa progression or deaths being described. These results do seem better compared to recent post-whole-gland treatment sRP series showing approximately 50\% PSM and BCR rates [11, 38] and not inferior to a primary RP setting [26]. Of note, one series reported almost one in two men having positive margins [24]. These results may reflect a suboptimal surgical expertise of 
some of the involved operators and strengthens EAU guidelines recommendations that sRP, even after FT, should be reserved to experienced surgeons only [39].

On the one hand, aggressive histology and advanced local disease (>pT3a) remained relatively rare in the post FT scenario compared to surgery following whole-gland treatments and may explain the higher cancer control rates after FT. In this context pelvic lymphadenectomy was performed inconsistently with only one man having N1 disease and its role remains unclear.

On the other hand, PCa failures were subject to significant upstaging/grading compared to before FT. Whether this is treatment-induced or due to disease under-estimation at first assessment remains to be determined. Nonetheless, in all three series, median time from first to salvage treatment was $<30$ months. Indeed, despite the absence of standardized criteria to define FT failure, a stringent follow-up after first-line treatment is key.

Possibly, increased use of mpMRI and implementation of other imaging modalities, including ${ }^{68} \mathrm{Ga}-$ PSMA-PET and/or the combination of both, may improve patient selection for FT and facilitate detection of recurrence and/or persistence at an early stage, avoiding disease mischaracterization and improving oncological outcomes [40-42].

Approximately $30 \%$ of the patients who were classified and treated with FT according to the organ confined disease had extracapsular extension at time of salvage surgery. Nonetheless, only four (7\%) had pT3b disease whilst the others had pT3a.

Further, some showed a higher BCR of post-FT sRP compared to a first line setting [26]. FT and/or delay in biopsy proven local recurrence depending on different institutional protocols may prolong definitive treatment significantly and alter the prognosis. FT follow up schedules certainly need to be standardized, claiming for international consensus. While the potential impact of diagnostic delays remains to be quantified, especially in light of evidence suggesting no impact of 6-months treatment delay, even in high-risk disease, close follow-up after FT is warranted [43].

Toxicity and functional recovery results of sRP are also encouraging. With the exception of erectile function preservation, and despite higher technical difficulty on the treated side, no marked differences are apparent compared to primary RP when the procedure is performed in experienced centres [26]. Excluding cases of previous bilateral FT, surgical complexity is not significantly altered as testified by short operating time and minimal blood loss. Similarly, complications are relatively rare yielding overall reasonable hospital stay and catheterization time. Continence is achieved by the majority of patients with severe incontinence being relatively rare. Interestingly, the sole study comparing sRP after whole- vs focal first-line treatment did not find any major differences with the exception of operating time, shorter for SRP after FT [25]. Absence of randomisation or statistical matching and the small study sample prompt further studies to overcome these limitations.

Two other salvage alternatives have been described, namely re-do ablation and SRT. Importantly, evidence derives almost entirely from post-whole-gland salvage series. Outcomes, especially in terms of functional recovery and comorbidities, may be different after FT. Limiting the treated area to a specific target and leaving un-modified benign surrounding areas produces fewer tissue alterations as confirmed by post-FT SRP series. Thus, less fibrosis and scarring may help to preserve key structures during first-line treatment, including the striated urethral sphincter and 
neurovascular-bundles, and theoretically lead to improved outcomes and safety profile following non-surgical salvage treatments.

Re-do ablation is feasible, yielding acceptable complications, oncological and functional outcomes. The sole study investigating whole-gland salvage cryotherapy after FT yielded acceptable cancer control although patient baseline features included some high-risk cases that would not have been ideal candidates for first-line FT [18]. Continence and erectile function were almost unchanged and no major complications were described, opposed to salvage cryo-ablation following wholegland BT and/or RT where, despite being uncommon, fistulas and strictures did still occur. No complication differences were highlighted between whole-gland re-do and first-line HIFU. Furthermore, the re-do group had shorter treatment time, catheterization and hospital stay [20, 21]. As highlighted by some authors, improvement of learning curve and technology are likely to play an important role which needs to be addressed in the coming years [19].

Focal salvage re-do ablation is also a viable solution for suitable candidates, with preliminary results showing non-inferior cancer control but improved OS compared to whole-gland approaches. As per first-line FT, focal salvage techniques may further reduce salvage treatmentrelated side effects [19].

Salvage radiotherapy is another feasible way to deal with FT failure as derived from post-whole gland-ablation series. Disease control is promising as are treatment-related toxicities which seem similar [29] or only slightly increased [37] compared to exclusive RT. However, due to the limited follow-up, approximately three years in the majority of published series, late complications remain to be determined.

On the contrary, as biochemical relapse after RT mainly presents within the third year after radiation $[28,44]$, oncological efficacy can be expected to be relatively high on longer follow-up despite these outcomes not being available yet.

Amongst the several aspects requiring further clarifications is the optimal total dose used in a post-FT setting as several series used a median of less than $72 \mathrm{~Gy}[27,28,31,35]$. Whilst this may not be sufficient in post-WT from an oncological perspective, it may as well reduce the burden of treatment related side effects. Again, no evidence is present following FT.

While the role of active surveillance, watchful waiting and ADT after FT were not assessed in our work, clinicians should consider these possibilities whenever appropriate[16, 17]. Certainly, in view of the different options and the poor evidence available, multidisciplinary decision-making is paramount in facing the challenge of FT failure [45].

Overall, although it does not allow any definitive conclusion, current evidence suggests the hypothesis that FT has minimal impact on subsequent salvage treatments, still allowing acceptable oncological success with minimal change in functional outcomes and complications. If confirmed, this would sustain FT development and introduction in clinical practice.

Nonetheless, high-quality studies are urgently needed to clearly determine many aspects of the impact of FT on subsequent salvage treatments and to optimize the use of FT per se and the management of FT failures.

The experience of Lebdai and colleagues, showing increased difficulty after bilateral versus unilateral VTP [24], favors the intuitive hypothesis that the more limited is the initial treatment, the more feasible is salvage surgery with neuro-vascular bundle preservation more likely to be 
performed. This is a key point as FT with margins $>/=8 \mathrm{~mm}$ is recommended but the more extensive the treatment delivery, the more challenging is salvage treatment, when indicated.

The impact of different energy sources and focal techniques on the subsequent salvage option needs to be clarified; whether one salvage treatment modality or focal technique proves superior compared to others may influence the choice of primary FT approach.

Also, the optimal salvage option is far from resolved as comparison amongst different treatments is not feasible without significant selection bias: these include lower PCa risk profile for those undergoing re-do ablation rather than surgery or radiotherapy and vice-versa. In some series, poor surgical candidates underwent whole-gland ablation due to greater comorbidities [37]. This is in distinction from the goal of FT which should represent an alternative for potential candidates for surgery or radiotherapy with the aim of decreasing morbidity and $[3,46]$. The same holds true for the absence of universally accepted criteria for FT and for definition of recurrence and/or failure. As a consequence, re-biopsies are mainly performed ad hoc rather than based on uniform predefined triggers. These should be entailed in a clear follow up schedule including a combination of imaging, biomarkers and patients' risk profile.

Finally, the most important point is we are not aware with the nature and natural history of PCa recurrence as FT is still in its early infancy.

Different energy types act and consequently may tend to fail differently. In prostate and breast cancer, surgery fails at the edges whilst radiotherapy tends to fail in the centre of the cancer. Similarly, some FTs may mimic surgery, others radiation.

In-/edge-of-field recurrence may be more likely to occur in the first years after FT, as in breast lumpectomy [47], usually due to incomplete cancer eradication and/or insufficient margins. After that time, in-/edge-of-field recurrence likelihood may then decrease, diverting the attention to the untreated benign prostatic tissue where untreated or new PCa microfoci may meet the threshold for clinical detection creating a new index lesion with different biological background compared to the earlier treated PCa.

In this context, FT may provide a unique "in-vivo" model of PCa progression in "clinically significant PCa formers" that has not been available to us by using whole gland treatments.

Currently, a UK-based prospective study, the RAFT trial (NCT03011606), is enrolling men undergoing robotic salvage surgery following FT failure. Results of this and other high-quality large studies are urgently needed and should be encouraged to fill the information gaps, optimizing management of FT failures, and enhancing our understanding of the impact of FT on subsequent salvage treatments.

\section{Conclusions}

Evidence assessing salvage treatments after FT failure is low, deriving from four retrospective salvage series post-FT and from post-whole-gland ablation or RT salvage series. Available salvage options include RP, re-do whole-gland and focal ablation and RT. Overall oncological outcomes are acceptable although biochemical recurrence is slightly increased compared to primary $\mathrm{PCa}$ 
treatment probably due to higher aggressiveness of recurrent/persistent PCa. Functional outcomes and complications are slightly worse but not markedly so compared to primary treatment. This favors the hypothesis FT may only marginally jeopardize subsequent salvage therapy. Future prospective high-quality studies are needed to specifically address the impact of FT on subsequent salvage treatments and to optimize the decision-making concerning FT failures.

\section{Acknowledgements}

Mark Emberton receives research support from the United Kingdom's National Institute of Health Research (NIHR) UCLH/UCL Biomedical Research Centre. He has held an NIHR Senior Investigator Award since 2013.

\section{Figure and Tables Legend}

Figure 1. Treatment Options for Persistent/Recurrent Prostate Cancer after Focal Therapy for Prostate Cancer. FT=Focal Therapy; $\mathrm{PCa}=$ Prostate Cancer.

Table 1. Main Series Reporting Salvage Cryotherapy and/or HIFU following Prostate Cancer recurrence after Focal Therapy or after whole-gland treatments including whole-gland ablations. $\mathrm{F}$ to $\mathrm{S}$ treatment=time from first to salvage treatment, months; ${ }^{*}=$ mean (interquartile range); ${ }^{\wedge}=$ subgroup receiving focal salvage cryosurgery; ${ }^{\wedge}=n=27$ ultimately had $B C R$ including those $n=11$ men who had 2 salvage cryotherapy cycles; '=median (range); " or ${ }^{\circ}=$ mean (range); PCa=prostate cancer.

Table 2. Main Series Reporting Salvage Radical Prostatectomy Outcomes for recurrent Prostate Cancer after Focal Therapy. $F$ to $S$ treatment=time from first to salvage treatment, months; $\mathrm{PCa}=$ prostate cancer ; $\mathrm{R} 1=$ positive surgical margins; "=values showed as median (IQ range); ${ }^{\circ \circ}=$ values showed as mean (SD); ${ }^{\circ}=$ mean (range); ${ }^{* \circ}=$ data for primary RARP are reported in the salvage treatment section to simplify data comparison.

Complications onset timings were rarely available in the three series; Lebdai et al: $\mathrm{n}=1$ man had a proximal urethral stenosis complicated with two episodes of urinary infections 1 month after the procedure; Linares et al: $n=13$ complications within $\leq 30$ days - in $n=9$ patients, no late complications. Information concerning erectile function changes of pre-operatively potent patients undergoing nerve sparing procedures was not always available; Linares et al: of preoperatively potent patients $(40 \%)$, at 12 months: $n=2(20 \%)$ had spontaneous or PDE-5I assisted sexual intercourse; $n=5$ (50\%) PGE or vacuum assisted erections; Nunes-Silva et al: data in the Table are reported according to potent patients undergoing nerve sparing procedures.

Table 3. Main Series Reporting the role of Salvage Radiotherapy for Prostate Cancer recurrence after local ablative HIFU and/or Cryotherapy. T to SRT= time to salvage radiation therapy; salvage $\mathrm{T}=$ salvage $\mathrm{RT}$ modality; $\mathrm{GS}=$ Gleason Score; $\mathrm{cT}=$ clinical $\mathrm{T}$ stage prior to $\mathrm{sRT}$; $\mathrm{BCR}=$ biochemical recurrence; $C S S=$ Cancer specific survival; $O S=$ overall survival; Urinary $F=$ Urinary Function; $E F=$ erectile function; $\mathrm{R}=$ retrospective; $\mathrm{CS}$ = cryosurgery; HIFU= High Intensity Focused Ultrasound; $\mathrm{ED}=$ 
erectile dysfunction; IG-IMRT=dose-escalated image guided intensity modulated radiation therapy; EBRT=external beam radiation therapy; SBRT=stereotactic body radiation therapy; '=median (range); "=mean (range); ${ }^{*}=\mathrm{n}=3$ patients received radiotherapy as an adjuvant treatment after cryotherapy; $\S=$ Biochemical (PSA) failure was defined according to the RTOGAmerican Society for Therapeutic Radiology and Oncology 2006 consensus definition; **=proton therapy; ${ }^{\wedge \wedge}=$ assessed on 32 patients; $\$=R T$ without ADT; NA=not available; ${ }^{\wedge}=$ at primary $C S$; ${ }^{*}=\mathrm{n}=3$ patients received neoadjuvant $\mathrm{RT} ;{ }^{\wedge \wedge \wedge}=$ not able to define if reported values were pre-first line treatment or pre-sRT; $\S \S=$ feasibility study of Riviere et al (patients included in the Riviere et al study).

\section{References}

[1] Valerio M, Ahmed HU, Emberton M, et al. The Role of Focal Therapy in the Management of Localised Prostate Cancer: A Systematic Review. European urology. 2013 Jun 6:

[2] Guillaumier S, Peters M, Arya M, et al. A Multicentre Study of 5-year Outcomes Following Focal Therapy in Treating Clinically Significant Nonmetastatic Prostate Cancer. European urology. 2018 Jun 27:

[3] Marra G, Gontero P, Valerio M. Changing the prostate cancer management pathway: why Focal Therapy is a step forward. Archivos espanoles de urologia. $2016 \mathrm{Jul}$ : 69:271-80

[4] Valerio M, Cerantola Y, Eggener SE, et al. New and Established Technology in Focal Ablation of the Prostate: A Systematic Review. European urology. 2016 Aug 29:

[5] van der Poel HG, van den Bergh RCN, Briers E, et al. Focal Therapy in Primary Localised Prostate Cancer: The European Association of Urology Position in 2018. European urology. 2018 Jan 17:

[6] Marra G, Ploussard G, Futterer J, Valerio M, Party E-YPCW. Controversies in MR targeted biopsy: alone or combined, cognitive versus software-based fusion, transrectal versus transperineal approach? World journal of urology. 2019 Jan 4:

[7] Marra G, Ploussard G, Ost P, et al. Focal therapy in localised prostate cancer: Real-world urological perspective explored in a cross-sectional European survey. Urologic oncology. 2018 Oct 6:

[8] Heidenreich A, Thuer D, Pfister D. Salvage radical prostatectomy. Panminerva medica. 2010 Sep: 52:231-8

[9] Heidenreich A, Richter S, Thuer D, Pfister D. Prognostic parameters, complications, and oncologic and functional outcome of salvage radical prostatectomy for locally recurrent prostate cancer after 21st-century radiotherapy. European urology. 2010 Mar: 57:437-43

[10] Chade DC, Eastham J, Graefen M, et al. Cancer control and functional outcomes of salvage radical prostatectomy for radiation-recurrent prostate cancer: a systematic review of the literature. European urology. 2012 May: 61:961-71

[11] Zargar H, Lamb AD, Rocco B, et al. Salvage robotic prostatectomy for radio recurrent prostate cancer: technical challenges and outcome analysis. Minerva urologica e nefrologica $=$ The Italian journal of urology and nephrology. 2017 Feb: 69:26-37

[12] Eandi JA, Link BA, Nelson RA, et al. Robotic assisted laparoscopic salvage prostatectomy for radiation resistant prostate cancer. The Journal of urology. 2010 Jan: 183:133-7

[13] Yuh B, Ruel N, Muldrew S, et al. Complications and outcomes of salvage robot-assisted radical prostatectomy: a single-institution experience. BJU international. 2014 May: 113:769-76 
[14] Dindo D, Demartines N, Clavien PA. Classification of surgical complications: a new proposal with evaluation in a cohort of 6336 patients and results of a survey. Annals of surgery. 2004 Aug:

240:205-13

[15] Mitropoulos D, Artibani W, Graefen M, et al. Reporting and grading of complications after urologic surgical procedures: an ad hoc EAU guidelines panel assessment and recommendations. European urology. 2012 Feb: 61:341-9

[16] Hamid S, Guillaumier S, Shah T, Arya M, Ahmed HU. Prostate cancer recurrence after Focal Therapy: Treatment options. Archivos espanoles de urologia. 2016 Jul: 69:375-83

[17] Barret E, Harvey-Bryan KA, Sanchez-Salas R, Rozet F, Galiano M, Cathelineau X. How to diagnose and treat focal therapy failure and recurrence? Current opinion in urology. 2014 May:

24:241-6

[18] Chang X, Liu T, Zhang F, et al. Salvage cryosurgery for locally recurrent prostate cancer after primary cryotherapy. Int Urol Nephrol. 2015 Feb: 47:301-5

[19] Wenske S, Quarrier S, Katz AE. Salvage cryosurgery of the prostate for failure after primary radiotherapy or cryosurgery: long-term clinical, functional, and oncologic outcomes in a large cohort at a tertiary referral centre. European urology. $2013 \mathrm{Jul}$ : 64:1-7

[20] Berge V, Dickinson L, McCartan N, et al. Morbidity associated with primary high intensity focused ultrasound and redo high intensity focused ultrasound for localized prostate cancer. The Journal of urology. 2014 Jun: 191:1764-9

[21] Blana A, Rogenhofer S, Ganzer R, Wild PJ, Wieland WF, Walter B. Morbidity associated with repeated transrectal high-intensity focused ultrasound treatment of localized prostate cancer. World journal of urology. 2006 Nov: 24:585-90

[22] Van Son MJ, Peters M, Moerland MA, et al. Re-salvage MRI-guided Focal High-dose-rate Brachytherapy for Locally Recurrent Prostate Cancer. Cureus. 2018 Apr 5: 10:e2429

[23] Murray KS, Akin O, Coleman JA. Irreversible Electroporation for Prostate Cancer as Salvage Treatment Following Prior Radiation and Cryotherapy. Reviews in urology. 2017: 19:268-72

[24] Lebdai S, Villers A, Barret E, Nedelcu C, Bigot P, Azzouzi AR. Feasibility, safety, and efficacy of salvage radical prostatectomy after Tookad(R) Soluble focal treatment for localized prostate cancer. World journal of urology. $2015 \mathrm{Jul}$ : 33:965-71

[25] Linares Espinos E, Sanchez-Salas R, Sivaraman A, et al. Minimally Invasive Salvage Prostatectomy After Primary Radiation or Ablation Treatment. Urology. 2016 Aug: 94:111-6 [26] Nunes-Silva I, Barret E, Srougi V, et al. Effect of Prior Focal Therapy on Perioperative, Oncologic and Functional Outcomes of Salvage Robotic Assisted Radical Prostatectomy. The Journal of urology. 2017 Nov: 198:1069-76

[27] Pasticier G, Chapet O, Badet L, et al. Salvage radiotherapy after high-intensity focused ultrasound for localized prostate cancer: early clinical results. Urology. 2008 Dec: 72:1305-9

[28] Riviere J, Bernhard JC, Robert G, et al. Salvage radiotherapy after high-intensity focussed ultrasound for recurrent localised prostate cancer. European urology. 2010 Oct: 58:567-73

[29] Munoz F, Guarneri A, Botticella A, et al. Salvage external beam radiotherapy for recurrent prostate adenocarcinoma after high-intensity focused ultrasound as primary treatment. Urologia internationalis. 2013: 90:288-93

[30] Ripert T, Bayoud Y, Messaoudi R, et al. Salvage radiotherapy after high-intensity focused ultrasound treatment for localized prostate cancer: feasibility, tolerance and efficacy. Canadian Urological Association journal = Journal de l'Association des urologues du Canada. 2012 Oct: 6:E179-83

[31] Burton S, Brown DM, Colonias A, et al. Salvage radiotherapy for prostate cancer recurrence after cryosurgical ablation. Urology. 2000 Nov 1: 56:833-8 
[32] Hepel JT, MacAusland SG, Long JP, Wazer DE, DiPetrillo T. Intensity-modulated radiotherapy of the prostate after cryotherapy: initial experience. Urology. 2008 Dec: 72:1310-4; discussion 4

[33] Hopper AB, Sandhu APS, Parsons JK, Rose B, Einck JP. Salvage image guided radiation therapy to the prostate after cryotherapy failure. Advances in radiation oncology. 2018 Jan-Mar: 3:52-6

[34] Choi M, Kim CR, Hung AY. Salvage intensity-modulated radiation therapy for locally recurrent prostate cancer after cryotherapy. Clinical genitourinary cancer. 2013 Jun: 11:85-8 [35] McDonough MJ, Feldmeier JJ, Parsai I, Dobelbower RR, Jr., Selman SH. Salvage external beam radiotherapy for clinical failure after cryosurgery for prostate cancer. International journal of radiation oncology, biology, physics. 2001 Nov 1: 51:624-7

[36] Quarrier S, Katz A, Haas J. Treatment of prostate cancer local recurrence after whole-gland cryosurgery with frameless robotic stereotactic body radiotherapy: initial experience. Clinical genitourinary cancer. 2013 Jun: 11:89-93

[37] Holtzman AL, Hoppe BS, Letter HP, et al. Proton Therapy as Salvage Treatment for Local Relapse of Prostate Cancer Following Cryosurgery or High-Intensity Focused Ultrasound. International journal of radiation oncology, biology, physics. 2016 May 1: 95:465-71 [38] Marra G, Gontero P, Alessio P, et al. Is it worth to perform radical prostatectomy in a salvage setting? Results of a contemporary multicentre series of 395 cases. Eur Urol Suppl [39] Mottet N, Bellmunt J, Bolla M, et al. EAU-ESTRO-SIOG Guidelines on Prostate Cancer. Part 1: Screening, Diagnosis, and Local Treatment with Curative Intent. European urology. 2017 Apr: 71:618-29

[40] Perera M, Papa N, Christidis D, et al. Sensitivity, Specificity, and Predictors of Positive (68)Ga-Prostate-specific Membrane Antigen Positron Emission Tomography in Advanced Prostate Cancer: A Systematic Review and Meta-analysis. European urology. 2016 Dec: 70:926-37

[41] de Rooij M, Hamoen EH, Witjes JA, Barentsz JO, Rovers MM. Accuracy of Magnetic Resonance Imaging for Local Staging of Prostate Cancer: A Diagnostic Meta-analysis. European urology. 2016 Aug: 70:233-45

[42] Chen M, Zhang Q, Zhang C, et al. Combination of (68)Ga-PSMA PET/CT and multiparameter MRI improves the detection of clinically significant prostate cancer: a lesion by lesion analysis. Journal of nuclear medicine : official publication, Society of Nuclear Medicine. 2018 Dec 14: [43] Gupta N, Bivalacqua TJ, Han M, et al. Evaluating the Impact of Length of Time from Diagnosis to Surgery in Patients with Unfavorable Intermediate to Very High-Risk Clinically Localized Prostate Cancer. BJU international. 2018 Dec 20:

[44] Hanlon AL, Diratzouian H, Hanks GE. Posttreatment prostate-specific antigen nadir highly predictive of distant failure and death from prostate cancer. International journal of radiation oncology, biology, physics. 2002 Jun 1: 53:297-303

[45] Martinez-Pineiro L, Sanchez C, Caceres F. Definition and management of prostate-specific antigen recurrence after local therapy. BJU international. $2007 \mathrm{Jul}$ : 100 Suppl 2:32-5

[46] Donaldson IA, Alonzi R, Barratt D, et al. Focal therapy: patients, interventions, and outcomes--a report from a consensus meeting. European urology. 2015 Apr: 67:771-7

[47] Yu KD, Li S, Shao ZM. Different annual recurrence pattern between lumpectomy and mastectomy: implication for breast cancer surveillance after breast-conserving surgery. The oncologist. 2011: 16:1101-10 